\title{
John Tyndall (1820-1893) \\ und seine Beziehungen zu den Alpen und zur Schweiz
}

Werner Sackmann

\section{SUMMARY}

John Tyndall, the British physicist and scientist who died a hundred years ago (4 Dec. 1893) was a great admirer of Switzerland and of its high mountains. Throughout his life as a researcher he was fascinated by glaciers, by their shape and movement. This made him an enthusiastic pioneer of alpine mountaineering. Besides his important attempts to conquer the Matterhorn he made the first successful ascent of the Weisshorn. On the Belalp, opposite the Weisshorn, he built a home for his annual vacations. The residents of Naters made him an honorary citizen of their community. On the Belalp, his widow erected a monument to remember Tyndall's affection for Switzerland and for the Alps.

\section{ZuSAMmenfassung}

Der britische Physiker und Naturforscher John Tyndall, dessen Todestag sich zum 100. Mal jährt, hatte ein besonderes Verhältnis zur Schweiz und ihren Hochgebirgen. Gestalt und Funktion der Gletscher hatten früh das Interesse des Forschers geweckt, so dass er ein begeisterter Bergsteiger und Alpenpionier wurde. Er zählt zu den frühesten Besteigern des Matterhorns und hat als erster das Weisshorn bezwungen. Auf Belalp, im Anblick des Weisshorns, baute er sich eine Ferienvilla. Dem mit Land und Leuten verbundenen Gast verlieh die Gemeinde Naters ihr Ehrenbürgerrecht. Ein Gedenkstein auf Belalp, den die Witwe zu seiner Erinnerung gestiftet hat, zeugt noch heute von der Verbundenheit Tyndalls mit der Schweiz.

Etwa acht Kilometer nördlich von Naters und Brig im Kanton Wallis erstreckt sich über den Südhang des Unterbächhorns und des Sparrhorns auf gut $2000 \mathrm{~m}$ Höhe die Belalp. Gemeinsam mit der im Osten gegenüberliegenden Riederalp überragt sie die Zunge des Aletschgletschers. Dem Berg- 
wanderer, welcher über die Belalp streift, fällt ein riesiger, granitener Megalith auf mit der folgenden Inschrift:

1820-1893

Raised to her all beloved by Louisa his wife to mark a place of memories

Diese ihm liebe Stätte widmet die Gemeinde Naters dem edlen englischen Naturforscher, ihrem Ehrenbürger.

Wie die Namensinschrift auf der Rückseite verrät, erinnert das Monument an den bedeutenden britischen Physiker John Tyndall und an dessen langjährige, tiefe Verbundenheit mit der Gegend. Seiner Liebe zu den Alpen und zur Schweiz sei diese Studie auf seinen 100. Todestag (4.Dezember 1993) gewidmet. Die Vorarbeiten dazu gehen zurück auf Peter Rudolf Merz (19281975), Chemiker in Visp, welcher in den 60er Jahren bereits eine Menge Daten zusammengetragen und bereitgestellt hat. Nach seinem Tode blieb das Material zunächst ungenutzt, bis es mir die Witwe in dankenswerter Weise zur freien Verfügung überliess.

Eine umfassende Biographie Tyndalls zu wiederholen, ist nicht beabsichtigt. Auch an Tyndalls zahlreiche Verdienste um sozusagen alle Teilgebiete der Physik sei hier nur andeutungsweise erinnert. Immerhin sei das korrekte Geburtsdatum noch einmal unterstrichen. Es ist dies, den unmittelbaren britischen Quellen ${ }^{1,2,3}$ zufolge, der 2 . August $1820^{4,5}$ und nicht etwa der 21. August, wie viele deutsche Lexika und Autoren behaupten ${ }^{6,7,8,9}$; auch nicht der 20. August, wie die Neue Zürcher Zeitung in ihrem Nachruf vom 10. Dezember 1893 angibt.

Tyndall stammte aus bescheidenen Verhältnissen im südöstlichen Irland. Als gelernter Geometer wurde er Vermessungsingenieur im Eisenbahnbau und Lehrer an einem College in der südenglischen Grafschaft Hampshire, bis ihm seine Ersparnisse erlaubten, ein Universitätsstudium nachzuholen.

Von der Begeisterung eines Thomas Carlyle ${ }^{10}(1795-1881)$ angesteckt, entschied sich Tyndall für Deutschland, das «Land der Universitäten»" Gemeinsam mit einem Lehrerkollegen vom Queenwood College bezog er im Herbst 1848 die damals ausgesprochen kleine Hochschule in Marburg a.d. Lahn, welche eine besonders individuelle und intensive Ausbildung versprach. Auf dem Marburger Schloss hatte schon viel früher ein Vorfahr Zuflucht gefunden, nämlich der Glaubenskämpfer William Tyndale (gest. 
1536), bekannt durch seine erste Übertragung des Neuen Testaments ins Englische.

In Marburg betrieb Tyndall ein ergiebiges Studium in Mathematik bei Friedrich Stegmann (gest. 1891), Chemie (Robert Bunsen, 1811-1899) und Physik (Hermann Knoblauch ${ }^{12}$, 1820-1895). Er erwarb 1850 den Doktorgrad mit seiner Dissertation «Die Schraubenflächen mit geneigter Erzeugungslinie und die Gleichgewichtsbedingungen für solche Schrauben». Marburg wurde auch zum Ausgangspunkt für eine erste Begegnung mit der Schweiz und ihren Gebirgen. Im September 1849 reiste Tyndall nämlich über Heidelberg nach Basel und von dort weiter - seiner bescheidenen Mittel wegen zu Fuss - nach Zürich. Von Arth her erstieg er die Rigi und wanderte über Gotthard-, Furka- und Grimselpass nach Guttannen, Grindelwald, Lauterbrunnen, Interlaken, und über Thun, Bern und Solothurn nach Basel, um von da nach Marburg zurückzufahren.

Die Alpen, aus der Ferne erstmals begrüsst, hatten Tyndall überwältigend und bleibend beeindruckt; doch aus der Nähe konnte er ihnen zunächst nicht viel abgewinnen. Er zog den Gesamteindruck vor ${ }^{13}$. Dass ihn die Alpen jedoch nicht mehr aus ihrem Banne entliessen, beweist die zweite Reise, zu welcher es $1856 \mathrm{kam}$, d.h. sobald seine Laufbahn einigermassen gesichert und wirtschaftliche Sorgen behoben waren ${ }^{14}$.

Tyndalls Interesse an den Alpen war eigentlich ein Interesse an Gletschern und Gletschereis. Es entsprang seiner Beschäftigung mit dem Diamagnetismus, d.h. mit dem in allen Stoffen durch ein äusseres Magnetfeld induzierbaren Magnetismus ${ }^{15}$. Insbesondere studierte er das diesbezügliche Verhalten zahlreicher Stoffe unter erhöhtem Druck, so auch von Wasser in seinen verschiedenen Aggregatzuständen ${ }^{16}$. Ein kurzer Einblick in die Geschichte der Gletscherkunde ist daher angebracht - mindestens um das wissenschaftliche Umfeld zu umreissen, welchem Tyndall sich zu seiner Zeit gegenüber sah ${ }^{17,18,19,20}$.

Im Jahre 1854 war z. B. das Buch «Die Gletscher der Jetztzeit» von Albert Mousson (1805-1890) erschienen ${ }^{21}$; und was Grossbritannien im besonderen betraf, war die Gletscherkunde damals dominiert vom schottischen Geologen und Alpenpionier James David Forbes (1809-1868), dem Verfasser von "Travels through the Alps of Savoy» ${ }^{22}$. Forbes betrachtete wie vor ihm schon Louis César Bordier (1747-1802) ${ }^{23,24}$ und insbesondere der spätere Bischof von Annecy, Louis Rendu (1789-1859) - das Gletschereis als eine visköse Masse, welche unter ihrem Gewicht talwärts gleitet. Nach Louis Rendu ${ }^{25}$ und Louis Agassiz (1807-1873) ${ }^{26}$ ist der Eisstrom am schnellsten in 
der Mitte und an der Oberfläche, am langsamsten randständig und in der Tiefe.

Für Tyndall hingegen war der Festkörper Eis kristallin aufgebaut. 1842 hatte ja der Solothurner Naturforscher Franz Josef Hugi (1796-1855) die Kornstruktur des Gletschereises entdeckt ${ }^{27}$. Da der Gefrierpunkt bei zunehmendem Druck sinkt, schmelzen Schneeflocken und Eiskristalle unter Druckeinwirkung $\left(\right.$ James $^{28}$ und William ${ }^{29}$ Thomson $\left.{ }^{30}\right)$, d.h. praktisch schon unter ihrem Gewicht, an den gegenseitigen Berührungsflächen und vereinigen sich alsdann infolge der sog. Regelation ${ }^{31}$ zu einem grösseren Brocken. Die Struktur des sekundär gefrorenen Eises solcher Trennflächen enthält weniger Luftblasen, weil während der vorübergehenden Verflüssigung Luft entwichen ist. Das Trennflächeneis ist daher nicht mehr weiss, sondern bläulich («Blaubänder»), was äusserlich die typische Äderung des Gletschereises bedingt. Unterdruck bzw. Spannung wird nicht etwa durch plastische Streckung beantwortet, sondern vielmehr durch Bruch. Nicht Viskosität, Duktilität und Plastizität, sondern Bruch und Regelation wurden somit von Tyndall für die Verformung der Eismassen bei ihrer schwerebedingten Wanderung verantwortlich gemacht ${ }^{32}$.

Bei geradlinigem Verlauf des Eisstromes wandern die Eismassen in der Mitte des Gletschers am schnellsten. In jeder Biegung des Verlaufs verlegt sich jedoch die Linie schnellster Wanderung nach der konvexen Seite der Kurve, genau wie in einem wässrigen Strom. Sie ist also stärker geschwungen als der Verlauf des Gletschertals an sich.

Durch die unterschiedliche Wanderungsgeschwindigkeit des Eises entstehen Spannungen, welche stets zu Rissen und Spalten von typischem Verlauf führen. Beim Überwälzen über konvexe Kanten entstehen längs gerichtete Spannungen und quer verlaufende Spalten, währenddem das Zurückbleiben randständiger und das Vordrängen medianer Partien eine schräg nach vorwärts und innen gerichtete Spannung erzeugt. Im rechten Winkel zu derselben entstehen die Risse, welche somit schräg von untenaussen nach oben-innen verlaufen.

Analog zu den Spalten verlaufen auch die Blaubänder oder Venen, welche umgekehrt durch entsprechend gerichtete Druckwirkung und Kompression entstehen. Neben schräg und quer verlaufenden Bändern tritt auch längs, d.h. in Richtung der Gletscherwanderung, verlaufende, longitudinale Bänderung auf, entstanden durch gegenseitige Bedrängung zweier sich vereinigender Gletscher. Der quer zu den Eisströmen einwirkende Druck verursacht Bänderung, die wiederum quer zur Druckrichtung, d.h. in Richtung 
des Gletschers verläuft. Die Schichtung des Eises entsteht also ähnlich wie die von Tyndall ursprünglich studierte Schichtung des Schiefers, welche sich ebenso quer zur Druck- bzw. Schwererichtung einstellt ${ }^{33}$.

Zusammenfassend beurteilt der bescheidene Tyndall die Verdienste so: «The idea of semifluid motion belongs entirely to Rendu; the proof of the quicker central flow belongs in part to Rendu, but almost wholly to Agassiz and Forbes; the proof of the retardation of the bed belongs to Forbes alone; while the discovery of the locus of the point of maximum motion belongs, I suppose, to me.s ${ }^{34}$

Mehr als 30 Jahre vergingen nun, ohne dass Tyndall nicht einige Sommerwochen oder -monate in den Alpen zugebracht hätte. Im Juli und August 1857 nahm er glaziologische Vermessungen am Montblanc vor, dessen Gipfel er ein erstes Mal am 13. August bestieg. 1858 erfolgten eine zweite Montblanc-Besteigung sowie Touren aufs Eggishorn ${ }^{35}$ und aufs Finsteraarhorn ${ }^{36}$. Zweimal in derselben Woche erstieg er den Monte Rosa (Dufourspitze), das erste Mal begleitet von Auguste Balmat (1808-1862) ${ }^{37}$, dem früheren Gehilfen von Forbes, das zweite Mal (17. August) sogar alleine.

Auch im Sommer 1859 arbeitete Tyndall eine kurze Weile im Gebiet des Montblanc, auf dessen Gipfel er mit zehn Begleitern eine ganze Nacht verbrachte ${ }^{38,39}$. Von besonderer Bedeutung aber war seine erste Winterexpedition über Weihnachten 1859, deren Befunde zum Vergleich mit den bisherigen Sommerbeobachtungen bestimmt waren. Seit Horace Benedict de Saussure (1740-1799) und Louis Agassiz galten Gletscher zur Winterszeit als unbeweglich, was schon Balmat unter Forbes' Leitung ansatzweise widerlegt hatte. Über Paris, Mâcon und Genf erreichte Tyndall am Weihnachtstag Chamonix und erstieg am 27. Dezember die Hütte Montenvers, zusammen mit vier Trägern ${ }^{40}$ und den beiden bewährten Führern Joseph Tairraz und Edouard Simond. Die winterlichen Vermessungen in der Mer-de-Glace, wo Forbes bereits 1842-1845 Triangulationen vorgenommen hatte ${ }^{41}$, waren harte Arbeit. Am 29. Dezember stieg die Gruppe wieder ab nach Chamonix und am Neujahrstag 1860 war Tyndall zurück in London. Das seit Monaten vorbereitete Buch «The Glaciers of the Alps» ${ }^{42}$ wurde nun abgeschlossen und erschien im Juli 1860 bei Murray ${ }^{43}$ in London. Alle weiteren Bücher verlegte Tyndall indessen bei William Longman (1813-1877), einem Gründungsmitglied des britischen Alpine Club.

In einem Zustand geistiger und körperlicher Erschöpfung glaubte Tyndall den Alpen fortan entsagen zu müssen. Doch bald fiel er ins pure Gegenteil und überbot sich geradezu selber. Im Sommer 1860 beteiligte er 
sich erstmals an dem bereits drei Jahre währenden Kampf ums Matterhorn $(4478 \mathrm{~m})$, welches vordem als völlig unersteigbar galt, so z. B. im Urteil von Forbes (1843) und von John Ball (1818-1889 $\left.{ }^{44}\right)$. «Accessible or not, the Mont Cervin is assuredly a different sort of affair from Mont Blanc or Monte Rosa, or any other of the thousand and one summits which nature has kindly opened to man.» ${ }^{45}$ Tyndall war mit seinem Freunde Francis Vaughan Hawkins und zwei Begleitern, Christian Lauener (1826-1891) ${ }^{46}$ und Christian Kaufmann, aus dem Berner Oberland angerückt, indem er am 9. August den Übergang von Lauterbrunnen zum Aletschgletscher über das von ihm so benannte Lauwinenthor erzwang. Am 20. August finden wir ihn in Breuil, wo er mit Johann Joseph Benet ${ }^{47}$ (1824-1864) aus Lax bei Fiesch ${ }^{48}$ und dem Italiener Jean Jacques Carrel zum Matterhorn aufbricht. Nach unvergesslichem Aufstieg über den Liongrat erreichten die drei den Grossen Turm $(3960 \mathrm{~m})$. Es war der insgesamt fünfte Besteigungsversuch am Matterhorn.

1862 unternahm Tyndall seinen zweiten Angriff aufs Matterhorn, wiederum von Breuil aus. Gefährten auf diesem insgesamt 15. Versuch waren Benet und Anton Walter, dazu als Träger Carrels Sohn Jean Antoine (1829-1890) und dessen Vetter César, der «Bersagliere», welcher die mörderische Schlacht von Solferino (1859) überlebt hatte. Sie erreichten den südwestlichen Satellitgrat, welcher später den Namen Pic Tyndall (4258 m)

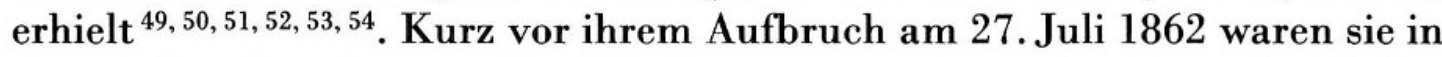
Breuil dem Landsmann Tyndalls begegnet, welcher den Berg drei Jahre später bezwingen sollte: Edward Whymper (1840-1911). Dieser kam in aller Munde sowohl dank seiner Pioniertat als auch wegen des tragischen Ausgangs derselben am 14. Juli $1865^{55}$. Im Abstieg stürzten bekanntlich vier der sieben Teilnehmer, drei Engländer und ein Führer, zu Tode ${ }^{56,57}$.

Noch im Juli 1865 kamen Tyndall im Haslital die ersten Gerüchte von Whympers Ungemach am Matterhorn zu Ohren. Von Mürren aus eilte er über die Gemmi nach Zermatt, wo er drei Wochen verblieb. Das schlechte Wetter erlaubte ihm indessen nicht, zur Unglücksstelle aufzusteigen, das letzte der vier Opfer (Lord Francis Douglas, 1847-1865) zu bergen ${ }^{69}$ und die Stelle durch Eisenhaken und Handseile für künftige Kletterer zu sichern.

Tyndall selber gelang die Besteigung des Matterhorns erst im Jahre 1868. Er erreichte, zusammen mit den Gebrüdern Maquignaz ${ }^{58}$ den Gipfel als siebenter ${ }^{59}$ seit Whymper. Julius M. Elliot († 1869 am Schreckhorn) war ihm gerade wenige Tage zuvorgekommen. Tyndalls Route zeichnete sich dadurch aus, dass er von der Südseite, also von Breuil her über den Liongrat aufstieg, um alsdann über den Hörnligrat nach Zermatt abzusteigen. 
Das Jahr 1861, zwischen den beiden unvollkommenen Matterhornbesteigungen, brachte einen um so schöneren Erfolg, nämlich die Erstbezwingung des Weisshorns, «an object scarcely less grand, conveying it may be even a deeper impression of majesty and might than the Matterhorn itself, ... perhaps the most splendid object in the Alps» ${ }^{60,61}$. Tyndall war am 4. August von Meiringen bzw. Hof durchs Urbachtal und über den Gauligletscher gestiegen und hielt sich einige Tage im Grimselgebiet auf. Am 13. August 1861 besuchte er erstmals die Belalp und das Sparrhorn (15. August), von wo er sein Traumziel ins Auge fassen konnte. Tags darauf begab er sich nach Randa im Mattertal. Am 19. August erreichte er aus einem Biwak am Schalliberggletscher (unterhalb des Ostgrates ${ }^{62,63}$ ) als erster zusammen mit J. J. Benet und Ulrich Wenger den Gipfel des Weisshorns (4512 m): «I had never before witnessed a scene which affected me like this one. I opened my note-book to make a few observations, but soon relinquished the attempt. There was something incongruous, if not profane, in allowing the scientific faculty to interfere where silent worship seemed the reasonable service.. ${ }^{64,65}$ Am 31. August 1861 berichtet das Walliser Wochenblatt : «Am 20.d. hat der Präsident ${ }^{66}$ des Londoner Alpenclubs mit vier ${ }^{67}$ Führern das Weisshorn zum ersten Mal erstiegen und wäre beinahe von herabstürzenden Gletscherblöcken erschlagen worden.» Gemeint ist wohl die Steinlawine, welche Tyndall schildert ${ }^{68}$.

Im Sommer 1869 gelang Tyndall die Bezwingung des Aletschhorns, welche er schon 1866 einmal versucht hatte. Einer anfänglich geringfügigen Sturzverletzung am Schienbein gewährte er anscheinend zu wenig Beachtung und Schonung. Jedenfalls entwickelte sich daraus eine schwere Sepsis mit Erysipel, Eiterung und Gangrän, was ihn zu mehrwöchigem Krankenlager bei englischen Freunden in Genf zwang. Er erlitt so am eigenen Leibe die Zusammenhänge zwischen Unsauberkeit und Eiterung, Infekt und Fieber, kurz zwischen «Dust and Disease» ${ }^{70,71}$, um einen seiner eigenen Titel und damit ein weiteres seiner Forschungsgebiete zu nennen, nämlich die mikrobielle Infektionslehre ${ }^{72}$ und die Frage nach der Urzeugung ${ }^{73}$.

Die Schweizer Aufenthalte der folgenden Jahre beschränkten sich nun mehr und mehr auf die geliebte Belalp und deren nähere Umgebung. Regelmässig logierte Tyndall in dem Berghotel, welches der unternehmungsfreudige Wirt Gervas Klingele 1860 erbaut hatte. 1876, am 11. Juli, erschien Tyndall dort erstmals in Gesellschaft seiner jungen Gattin Louisa Charlotte (1845-1940), Tochter des Lord Claud Hamilton (1813-1884), dem Bruder des ersten Herzogs (Duke) von Abercorn, und der Elizabeth Emma Proby 
(1821-1900), Schwester des dritten Earls of Carysfort. Das Paar, das sich seit etwa vier Jahren kannte, hatte sich anfangs 1876 in aller Stille vermählt; der Bräutigam war 56, die Braut 31 Jahre alt. Tyndall hatte zweifellos eine gute Partie gemacht, nicht nur in materieller Hinsicht. Er besiegelte damit seinen steilen gesellschaftlichen Aufstieg; er gewann aber auch eine selbstlose, besorgte Arbeitskameradin bei all seinen wissenschaftlichen Aktivitäten.

Die junge Ehe und der neue Wohlstand gaben zweifellos den Ausschlag zu folgendem Entschluss. «1876 den 13ten August zu Naters unter der Linde, nach gehöriger, vorausgegangener Publikation, versammelte sich unter dem Vorsitze des Burgerpräsidenten Johann Salzmann, die Burgergemeinde von Naters, welche mit achtundachzig ${ }^{74}$ Stimmen gegen sechs den Burgerrath oder ein Mitglied desselben ermächtiget und Vollmacht giebt dem Professor Johann Tyndall ein Stück Liegenschaft in der Alpe Lusgen unter nachstehenden Conditionen zu verkaufen und zu übertragen.» Unverzüglich begann der Maurermeister Sylvester Ramoni mit dem Bau der «höchstgelegenen Villa der Welt», und stellte sie auf November 1877 fertig.

Die Beziehungen Tyndalls zur Landschaft und zur Bevölkerung waren die denkbar besten. Besonders interessant, ja ergreifend sind die Schilderungen, wie er sich dank seiner Kenntnisse unentgeltlich als Arzt und Tierarzt betätigte auf der abgelegenen, von der Zivilisation entfernten Alp ${ }^{75}$. Man fühlte sich geehrt, einem so berühmten ausländischen Gelehrten Gastrecht gewähren zu dürfen.

Unter den vielen britischen und ausländischen Ehrungen, welche Tyndall als Akademiker, Alpinist und Forscher zuteil wurden, nennt die Biographie auch das Ehrenbürgerrecht der Gemeinde Naters. Dieses soll ihm 1887, nach dem Walliser Boten vom 23. September 1974 sogar schon 1885 zuerkannt worden sein. Doch geben die Biographen keinerlei weitere Hinweise oder Belege zu ihrer Angabe; auch fanden sich weder in der Tages- bzw. Wochenpresse ${ }^{76}$ jener Jahre noch im Archiv der Gemeindekanzlei Naters irgendwelche Dokumente ${ }^{77}$. Trotz dieser Sachlage wäre es indessen ungerecht, an der Zuerkennung des Ehrenbürgerrechtes zu zweifeln. Nur scheint es, dass dieselbe - wenn auch coram publico - rein mündlich, von Mann zu Mann, erfolgte und nicht protokolliert wurde, was in Anbetracht der zeit- und ortsbedingten Verhältnisse weiter nicht zu verwundern braucht. Abgesehen von Angelegenheiten mit finanziellen Konsequenzen wie etwa ein Kaufvertrag (vgl. oben) genügte meist das Wort des freien Mannes vollauf und bedurfte der schriftlichen Bekräftigung nicht. Auch war die Presse im Vergleich zu heute längst nicht so omni- und die übrigen Medien überhaupt 
noch nicht präsent. Eine Urkunde von besonderer Art gibt es jedoch: Auf dem eingangs schon beschriebenen Monolithen wurde rund 25 Jahre später das Zeugnis der Ehrenbürgerschaft in Stein gehauen!

Mit dem Jahr 1887 ging Tyndalls äussere berufliche Wirksamkeit zur Neige, indem er von seiner Professur an der Royal Institution zurücktrat. Mehr und mehr liess auch seine Gesundheit zu wünschen übrig, die ohnehin zeitlebens nie besonders robust gewesen war. Fast lebenslang litt Tyndall unter Indigestion und Schlaflosigkeit. Man wundert sich, wie er jeweils die anstrengenden, langen Fusswanderungen und Klettertouren bewältigte, wobei er sich allerdings nie wohler und leistungsfähiger fühlte als in der sommerlichen Schweiz.

Durch altersbedingte Hinfälligkeit gezwungen, sah Tyndall 1891 erstmals von einer Schweizerreise ab; doch fand er sich die beiden folgenden Sommer wieder auf Belalp ein. Nach seiner letzten Heimreise am 21. Oktober 1893 lebte er gerade noch sechs Wochen. Das Ende war nur mittelbar krankheitsbedingt; die unmittelbare Todesursache war begründet in der Vielzahl von Medikamenten, ohne die er nicht mehr auskam. Es handelte sich weder um Mord noch um Selbstmord; aber der geheimnisumwitterte Vorfall zog dennoch peinliche juridische Kontroversen nach sich.

Die Neue Zürcher Zeitung meldete am 6. Dezember 1893 ebenso lapidar wie unverblümt: «Der Tod des Professors Tyndall erfolgte, weil er Chloral verschluckte.» Schonender fiel die Todesnachricht des deutschen Alpenvereins aus: «Seine Gattin reichte ihm die gewöhnliche Dosis Chloral, seine Schwäche war aber schon so gross, dass er, wie man annimmt, das Medicament nicht vertragen konnte. ${ }^{78}$ Genau genommen wollte es die Tragik, dass Tyndalls Frau in glaubhaft unbeabsichtigter Weise zwei Medikamente verwechselte. Anstelle des Antazidums Magnesiumkarbonat (Magnesia) löste sie eine gleiche Menge des Beruhigungsmittels Chloralhydrat auf, was auch für einen Gesunden eine Überdosis darstellt ${ }^{79}, 80$. Sie hatte noch nahezu 47 Jahre unter der Last dieses Unglücksfalles zu leben, denn sie folgte ihrem Gatten erst 1940, also im hohen Alter von 95 Jahren, im Tode nach.

Die tiefe Zuneigung zu ihrem unvergesslichen Gatten, die Schuldgefühle wegen seines tragischen Endes und die Verbundenheit mit Land und Leuten dürften die Beweggründe gewesen sein für das steinerne Erinnerungsmal, welches die Jahr für Jahr nach Belalp pilgernde Witwe aus einem Findling der näheren Umgebung gestalten liess.

Zur offiziellen Einweihung lud Gemeindepräsident Meinrad Michlig auf Sonntag, den 27. August 1911 ein $^{81}$. Weil die Gemeinderäte am Vormittag 
unter der Linde in Naters noch einer wichtigen Gemeindeversammlung vorzustehen hatten, konnten sie erst in der Nachmittagshitze nach Blatten und zur Belalp aufsteigen ${ }^{82}$. Dass man die Feier deshalb erst um $19 \mathrm{Uhr}$ beginnen konnte, tat ihrer Würde keinen Abbruch, im Gegenteil: «Ein wunderbar herrlicher Abend senkte sich auf die Berge herab, die höchsten Spitzen flimmerten noch im letzten Sonnengold, während der Talgrund bereits in Dämmerung sich hüllte. Ringsum herrschte Schweigen und majestätische Ruhe. Da fand droben auf dem Trimbiel, auf einem aussichtsreichen Hügel eine halbe Stunde ob Bel-Alp, eine schlichte aber doch schöne und würdige Feier statt. Es wurde nämlich das Denkmal des berühmten englischen Naturforschers J. Tyndall der Obhut der Gemeinde Naters übertragen.» ${ }^{83}$ Nach der Eröffnung durch den Gemeindepräsidenten sprachen nacheinander Pfarrer Dionys Imesch ${ }^{84}$ von Naters, Oberst Douglas James Proby late Hamilton (1856-1931) ${ }^{85}$ als Vertreter der Stifterfamilie, Edouard Sarasin ${ }^{86}$, Präsident der Schweiz. Naturforsch. Gesellschaft, und Vikar Bamatter als Inhaber der Kaplanei Belalp. Anschliessend lud der Gemeindevorsteher zu einem Bankett im Hotel Belalp und zu einem Volksfest, an dem auch ein vom Schweizer Alpenclub gestiftetes Feuerwerk hochging ${ }^{87}$.

Ob der Einweihungstag zufällig oder absichtlich so gewählt worden war, bleibe dahingestellt; jedenfalls hatte sich soeben Tyndalls Erstbesteigung des Weisshorns (19.August 1861) zum 50. Mal gejährt. Und noch wieder rund 50 Jahre später gedachte der greise Gemeindepräsident der Feier von 1911 im Walliser Boten vom 7. August 1964. Auf den 23. Januar 1964 hatte nämlich Frau Tyndalls Neffe, Major Richard George Proby (1886-?), 1st Baronet of Carysfort, die Villa Tyndall an einen Basler Zahnarzt verkauft. Nach wie vor aber steht «droben auf Belalp ein Denkmal, einfach und schlicht und doch erhaben und imposant, das auch späteren Geschlechtern Kunde bringt von dem berühmten englischen Naturforscher und Alpenfreund John Tyndall» ${ }^{88}$, von seinen Beziehungen zu den Bergen und zur Schweiz. 


\section{Literatur und Anmerkungen}

Encyclopedia Britannica 15th ed., 1982.

Dictionary of National Biography; London: Smith, Elder \& Co., 1899.

3 Dictionary of scientific biography (Ch. C. Gillispie ed.); New York: Scribner, 1976.

4 Hermann, A.: Geschichte der Physik; Köln: Deubner, 1972.

5 Handwörterbuch der Naturwissenschaften (R. Dittler ed.) Bd. 10; Jena : Fischer, 1935.

6 Poggendorff, J. C.: Biographisch-literarisches Handwörterbuch zur Geschichte der exakten Wissenschaften; Leipzig: Barth, 1863, 1897, 1904.

7 Leopoldina $29: 210-211$ (1893).

8 Haeberlin, C.: John Tyndall; Leopoldina 30 : 94-97, 114-120, 130-138 (1894).

9 Brockhaus Enzyklopädie 17. Aufl., Bd. 19; Wiesbaden: Brockhaus, 1974.

10 Er war Tyndalls Trauzeuge am 29. Februar 1876.

11 s. 8

12 Auf ihn dürfte Tyndalls Interesse am Diamagnetismus zurückgehen.

13 Rossel, A.: John Tyndall et Henri Hansjakob; Le Petit Jurassien (Moutier) 1913.

$14 . \quad$ s. 3

15 Tyndall, J. \& Knoblauch, H.: The development of crystalline bodies between the poles of a magnet; Phil. Mag. 37 : 1-33 (1850).

16 Tyndall, J. : Forms of Water in clouds, rivers, ice and glaciers; London: King, 1872.

17 Rütimeyer L.: Ein Blick auf die Geschichte der Gletscherstudien in der Schweiz; Jahrb. Schweiz. Alpenclub 16: 377-418 (1881).

18 Heim, A.: Handbuch der Gletscherkunde (VI. Die Theorie der Gletscherbewegung, p. 284337); Stuttgart: Engelhorn, 1885.

19 v. Drygalski, E. \& Machatschek, F. : Enzyklopädie der Erdkunde, Teil 3: Gletscherkunde; Wien: Deuticke, 1942.

20 Wilhelm, F.: Schnee- und Gletscherkunde (Lehrbuch d. allgem. Geographie, Bd. 3, Teil 3); Berlin-New York: De Gruyter, 1975.

21 Mousson, A.: Die Gletscher der Jetztzeit ; Zürich: Schulthess, 1854.

22 Forbes, J. D.: Travels through the Alps of Savoy; Edinburgh : Black, 1843.

23 Bordier, L.C.: Voyage pittoresque aux glacières de Savoye fait en 1772; Genève 1773.

24 Senebier J., Hist. litt. de Genève III : 297; Genf 1786.

25 Rendu, L.: Théorie des glaciers de la Savoie; Chambéry 1840.

26 Agassiz, L.: Système glaciaire; Paris: Masson, 1847.

27 Hugi, F. J.: Über das Wesen der Gletscher und Winterreise in das Eismeer; StuttgartTübingen: Cotta, 1842.

$28 \quad 1822-1892$

29 1824-1907, seit 1866 Lord Kelvin of Largs.

30 Thomson, J.: Theoretical considerations on the effect of pressure in lowering the freezing point of water; Trans. Roy. Soc. Edinb. 16: 575-580 (1849).

31 Ausdruck geprägt von Joseph Dalton Hooker; s. 38 (p. 280).

32 Dieser Streit blieb anscheinend noch lange unentschieden; vgl. Seligman, G.: Forschungsergebnisse am grossen Aletschgletscher; Die Alpen 19 : 357-364 (1943).

33 Tyndall, J.: Comparative view of the cleavage of crystals and slate rocks; Roy. Inst. Proc. II : 295-308 (1854-1858). 
34 Tyndall, J.: The glaciers of the Alps; London: Murray, 1860 (p. 310).

35 30. Juli 1858 = erste von zahlreichen Eintragungen im Journal des Hotels Eggishorn (Seylaz, L.: Rund ums Eggishorn; Die Alpen 34 : 51-60 [1958]).

36 Lehner, W.: Die Eroberung der Alpen; Leipzig-Zürich: Grethlein, 1924.

37 s. 23

38 Eve, A.S. and Creasey, C. H.: Life and work of John Tyndall; London: Macmillan, 1945 (p. 360-361).

39 Tyndall, J. : Hours of exercise on the Alps; London: Longmans, Green \& Co., 1871 (p. 57).

40 Darunter Edouard Balmat («le petit B.»).

41 s. 20

42 s. 34

43 Gründer des Verlags war der vormalige Marineleutnant John Murray (1778-1843); «Guide Murray» = Handbook for travellers in Switzerland and in the Alps of Savoy, 1854/56.

44 Ball, J.: The Alpine Guide; London: Longmans, Green \& Co., 1863.

45 s. 39 (p. 30)

46 Egger, K.: Pioniere der Alpen; Zürich: Amstutz-Herdeg, 1946.

47 Von den Engländern beharrlich «Bennen» genannt; sein Bergtod auf dem Haut de Cry (2969 m), einem Diablerets-Vorgebirge, ist in 39, p. 192-205 beschrieben.

48 s. 46

49 Anon.: Matterhorn-Besteigungsversuche; Alpinismus (München), H. 6 (1965).

50 Gosset, Ch.: Tableau schématique des tentatives au Cervin de 1857 à 1865; Die Alpen 18 : 325-330 (1942).

51 Truffer, B. P.: Die Geschichte des Matterhorns; Zermatt: Aroleit, 1990.

52 Nünlist, H.: Matterhorn-Furggengrat; Die Alpen 22 : 161-167 (1946).

53 Anon.: John Tyndall und das Matterhorn; Walliser Bote-Briger Anzeiger, p. 4, 13. August 1970.

54 s. 36

55 Senger, M.: Wie die Schweizer Alpen erobert wurden; Zürich: Büchergilde Gutenberg, 1945.

56 Zsigmondy, E.: Die Gefahren der Alpen; Leipzig : Frohberg, 1885.

57 Anon.: 100 Jahre Matterhorn; Sonderdruck aus «Der Bergsteiger» 32. Jahrg., Juli 1965.

58 J. J. und Pierre, aus Valtournanche; sie waren schon 1867 (13. September) via Col Félicité erfolgreich am Matterhorn.

59 s. 38 (p.131)

60 Tyndall, J.: Mountaineering in 1861 : a vacation tour; London: Longmans, Green \& Co., 1862 (p. 25/26).

61 s.39 (p. 81)

62 Keenlyside, F.: Berge und Pioniere; Zürich : Orell Füssli, 1976.

63 Robert, E. A.: Au Weisshorn; Die Alpen, 6 : 307-314 (1908).

64 s. 39 (p. 106)

65 s. 60 (p. 58$)$

66 bestenfalls: Vizepräsident

67 Sie wurden von zwei Einheimischen verfolgt und beobachtet (vgl. 39, p. 189).

68 s. 39 (p. 110).

70 Tyndall, J.: Dust and disease; Roy. Inst. Proc. 6 : 1-14 (1870). 
71 Tyndall, J.: Fragments of science for unscientific people; New York: Appleton, 1873.

72 Tyndall, J.: Essay on the floating matter of the air in relation to putrefaction and disease; London: Longmans, Green \& Co., 1881.

73 Tyndall, J.: La génération spontanée; Revue scientifique 14 : 1197-1210 (1878).

74 sic!

75 Tyndall, J.: Leben in den Alpen (Fragmente, neue Folge); Braunschweig: Vieweg, 1895 (p.358).

76 «Walliser Bote» und «Briger Anzeiger».

77 Merz, P. R.: John Tyndall, eine kurze Biographie mit besonderer Berücksichtigung seiner Beziehungen zur Belalp und der Gemeinde Naters (Manuskript, o. J.).

78 Mitt.d. deutschen und österr. Alpenvereins 19: 293 (1893).

79 s. $38($ p. $279-280)$

80 s. 8

81 Briefkopie im Besitze von Hrn. M. Michlig jun., Naters.

82 Journal de Genève Nr. 243, 5. September 1911.

83 Briger Anzeiger Nr. 70, 1911.

84 Dr. theol. von Mörel, geb. 1868, Pfarrer von Naters 1903-1917, dann Domherr in Sitten.

85 Bruder von Mrs. Tyndall und Vetter des zweiten Duke of Abercorn; hatte 1904 den Familiennamen seiner Mutter (Proby) angenommen.

86 1843-1917, Physiker in Genf, Zentralpräsident der SNG 1911-1916.

87 Wallsier Bote, 2.September 1911.

88 Walliser Bote, 7. August 1964.

Dr. med. vet. W. Sackmann

St. Johanns-Parkweg 3

CH-4056 Basel 